\title{
QUALIDADE DA ASSISTÊNCIA E SEGURANÇA DO PACIENTE: AVALIAÇÃO POR INDICADORES
}

\author{
Daniela Patrícia Tres ${ }^{1}$, João Lucas Campos Oliveira ${ }^{2}$, Dagmar Willamowius Vituri ${ }^{3}$, Sidnei Roberto Alves ${ }^{4}$, \\ Denise de Fátima Hoffmann Rigo ${ }^{5}$, Anair Lazzari Nicola ${ }^{6}$
}

RESUMO: Estudo descritivo, transversal, prospectivo, observacional, quantitativo, que objetivou avaliar a qualidade da assistência com indicadores relacionados à prevenção de quedas e identificação do paciente de um hospital universitário. Os dados foram coletados em 15 dias aleatórios dos meses de agosto e setembro de 2015, em duas unidades de um hospital do interior do Paraná-Brasil, utilizando-se instrumentos previamente validados. Fez-se análise estatística descritiva, seguido do cálculo do Índice de Positividade. Foram realizadas 1068 observações. A qualidade da assistência ilustrada pelos indicadores de identificação do paciente pelo leito/ pulseira e presença de grades no leito à prevenção de quedas foi classificada como sofrível ( $0 \%)$, limítrofe $(76,60 \%)$, e adequada $(95,48 \%)$, respectivamente. Concluiu-se a necessidade iminente de melhorias na identificação do paciente, o que possivelmente poderia ser viabilizado pela escolha racional do método de identificação e através do empoderamento do enfermeiro em relação à tomada de decisão quanto a este indicador.

DESCRITORES: Qualidade da assistência à saúde; Segurança do paciente; Indicador de qualidade; Enfermagem; Avaliação em saúde.

\section{QUALITY OF CARE AND PATIENT SAFETY: ASSESSMENT BASED ON INDICATORS}

\begin{abstract}
Descriptive, cross-sectional, prospective, observational, quantitative study that was aimed at assessing the quality of care, using indicators related to falls prevention and patient identification at a teaching hospital. The data were collected on 15 random days in August and September 2015 at two services of a hospital in the interior of Paraná-Brazil, using previously validated tools. Descriptive statistical analysis was applied, followed by the calculation of the Positivity Ratio. In total, 1068 observations were made. The quality of care, illustrated by the indicators of patient identification by the bed/wristband and presence of bedrails to prevent fall was classified as low $(0 \%)$, borderline $(76.60 \%)$ and appropriate $(95.48 \%)$, respectively. In conclusion, improvements in patient identification are imminently necessary, which could be made feasible by the rational choice of the identification method and through the empowerment of the nurse for decision making on this indicator.
\end{abstract}

DESCRIPTORS: Quality of health care; Patient safety; Quality indicator; Nursing; Health evaluation.

\section{CALIDAD DE LA ATENCIÓN Y SEGURIDAD DEL PACIENTE: EVALUACIÓN POR INDICADORES}

RESUMEN: Estudio descriptivo, trasversal, prospectivo, observacional, cuantitativo, con objeto de evaluar la calidad de la atención con indicadores relacionados a la prevención de caídas e identificación del paciente de un hospital universitario. Los datos fueron recolectados en 15 días aleatorios de los meses de agosto y septiembre del 2015, en dos unidades de un hospital del interior de Paraná-Brasil, utilizándose instrumentos previamente validados. Fue aplicado análisis estadístico descriptivo, seguido del cálculo del Índice de Positividad. Fueron efectuadas 1068 observaciones. La calidad de la atención ilustrada por los indicadores de identificación del paciente por el lecho/la pulsera y presencia de grades en el lecho para prevención de caídas fue clasificada como sufrible $(0 \%)$, limítrofe $(76,60 \%)$ y adecuada $(95,48 \%)$, respectivamente. Se concluyó la necesidad inminente de mejoras en la identificación del paciente, lo que posiblemente podría ser viabilizado por la elección racional del método de identificación y a través delempoderamiento del enfermero con relación a la toma de decisión respecto a este indicador.

DESCRIPTORES: Calidad de la atención de salud; Seguridad del paciente; Indicador de calidad; Enfermería; Evaluación en salud.

${ }^{1}$ Enfermeira. Especialista em Gerenciamento de Enfermagem em Clínica Médica e Cirúrgica. Universidade Paranaense. Cascavel, PR, Brasil.

${ }^{2}$ Enfermeiro. Doutorando em enfermagem. Docente de Enfermagem e do Programa de Residência em Gerenciamento de Enfermagem em Clínica Médica e Cirúrgica da Universidade Estadual do Oeste do Paraná. Cascavel, PR, Brasil.

${ }^{3}$ Enfermeira. Doutora em Enfermagem. Hospital Universitário da Universidade Estadual de Londrina. Londrina, PR, Brasil.

${ }^{4}$ Enfermeiro. Mestre em Promoção da Saúde. Hospital Universitário do Oeste do Paraná. Cascavel, PR, Brasil.

${ }^{5}$ Enfermeira. Pós-graduanda do Programa de Residência em Gerenciamento de Enfermagem em Clínica Médica e Cirúrgica. Hospital Universitário do Oeste do Paraná. Cascavel, PR, Brasil.

${ }^{6}$ Enfermeira. Doutora em Enfermagem. Docente de Enfermagem e coordenadora do Programa de Residência em Gerenciamento de Enfermagem em Clínica Médica e Cirúrgica da Universidade Estadual do Oeste do Paraná. Cascavel, PR, Brasil.

Autor Correspondente:

Recebido: 02/02/2016

Daniela Patrícia Tres

Universidade Estadual do Oeste do Paraná

Finalizado: 03/07/2016

R. Universitária, 1619 - 85819-110 - Cascavel, PR, Brasil

E-mail: danielapatriciatres@gmail.com 


\section{INTRODUÇÃO}

Em vista, principalmente, do avanço científico-tecnológico acelerado; do aumento das exigências dos consumidores; e da crescente competitividade no mundo globalizado hodierno, as organizações prestadoras de serviços, ao exemplo das instituições de saúde, veem-se cada vez mais na necessidade de garantir a qualidade da assistência prestada. Nesta direção, alguns serviços de saúde vêm incorporando os princípios clássicos da qualidade à luz gerencial, especialmente no que tange à busca da melhoria da assistência, por meio de ações sistemáticas e cíclicas de avaliação(1).

Apesar de a avaliação ser uma prática de gestão elementar a favor do cuidado qualificado, a qualidade na saúde é um bem complexo e polissêmico, regida por bens que ultrapassam a sua garantia viabilizada unicamente pelos processos avaliativos, ao exemplo da eficácia, eficiência, acessibilidade, aceitabilidade, equidade, e, mais recentemente, segurança ${ }^{(2)}$.

A segurança não significa garantia de cuidado integralmente qualificado, todavia, é um dos pilares que alicerça a qualidade na saúde, visto que os riscos associados ao atendimento neste peculiar setor de produção são evidentes ${ }^{(2,3)}$. Neste aspecto, a segurança do paciente é definida como a redução do risco de danos desnecessários associados à atenção à saúde, até um mínimo aceitável, pois, considerandose a complexidade de procedimentos e tratamentos incutidos nos serviços prestadores de assistência, o potencial para o dano ao paciente é real ${ }^{(4)}$.

As práticas que visam a favorecer o atendimento seguro ao paciente são diversas, indo desde a promoção da cultura organizacional favorável a este bem $^{(3)}$, até o estabelecimento de metas, medidas e protocolos pontuais com a finalidade de reduzir os riscos associados ao cuidado ${ }^{(4)}$. Destarte, a prevenção da queda durante a hospitalização, bem como a identificação do paciente, estão entre as estratégias para redução dos incidentes de segurança, sendo algumas das metas estabelecidas pelo Programa Nacional de Segurança do Paciente (PNSP) ${ }^{(4)}$.

As quedas sofridas pelos pacientes durante a internação hospitalar são uma das ocorrências mais importantes na quebra da segurança no atendimento, e são frequentemente responsáveis pelo aumento do número de dias de internamento e piores condições de recuperação ${ }^{(5)}$. Isso porque a ocorrência da queda no ambiente hospitalar tem potencial para agravar os problemas de saúde do paciente, e as principais consequências são: traumas; retirada não programada de cateteres, drenos e sondas; medo de cair novamente; alterações de ordem emocional; piora clínica; e até mesmo o óbito ${ }^{(6)}$.

Outra prática que merece atenção à promoção da segurança no cuidado éa identificação do paciente, que é abrangente e de responsabilidade multidisciplinar, uma vez que envolve aspectos de estrutura, desenhos dos processos de trabalho, cultura organizacional, prática profissional e participação do usuário $^{(7)}$. Portanto, postula-se que a não conformidade na identificação do paciente perfaz um fator preocupante na assistência à saúde, evidenciando que esta prática pode induzir a uma série de erros e até mesmo eventos adversos ${ }^{(8)}$.

Sob o entendimento de que a prevenção das quedas e a correta identificação do paciente são elementos que alicerçam a promoção do atendimento seguro(4), entende-se que o conhecimento do risco à queda pode significar um indicador para o monitoramento da segurança do paciente neste aspecto $^{(9,10)}$.

A avaliação da identificação do paciente também se posta como um indicador para o controle da qualidade do cuidado, que tem potencial para subsidiar a prevenção de erros e eventos adversos, e, assim, favorecer a assistência mais segura ${ }^{(11)}$. Isso é relevante porque os indicadores de qualidade se destacam como ferramentas/instrumentos gerenciais que subsidiam as práticas racionais de avaliação, o que favorece a tomada de decisão para a melhoria contínua ${ }^{(12)}$.

Este estudo justifica-se à seguinte prerrogativa: a análise de indicadores relacionados à qualidade e a segurança do paciente tem potencial para fundamentar o processo decisório, com a finalidade de subsidiar medidas que favoreçam o atendimento seguro.

Este fato pode ser melhor elucidado por meio da produção do conhecimento científico, em vista do seu potencial para (re)direcionar práticas de gestores e profissionais de saúde no que diz respeito 
à monitoração e avaliação cíclica de fatores que permeiam a qualidade no cuidado e a segurança do usuário.

Frente à problemática apresentada, surge o seguinte questionamento: Qual é qualidade da assistência relacionada à prevenção de quedas e identificação do paciente em um hospital de ensino público? A fim de responder esta indagação, este estudo objetivou avaliar a qualidade da assistência por meio dos indicadores relacionados à prevenção de quedas e identificação do paciente em um hospital de ensino público.

\section{- MÉTODO}

Trata-se de um estudo descritivo, transversal, prospectivo, observacional, de abordagem quantitativa. Foi desenvolvido em um hospital universitário público do interior do estado do Paraná, Brasil, com os pacientes internados nas unidades de Clínica Médica e Cirúrgica Geral (F2); e Neurologia, Ortopedia e Vascular (G3).

O hospital em questão possui 195 leitos, sendo o único hospital público da região oeste do Paraná, com $100 \%$ da capacidade operacional destinada ao Sistema Único de Saúde (SUS). Ademais, à época do estudo, as unidades estudadas possuíam 25 leitos na unidade que atende as especialidades ortopedia (12), neurologia (10) e vascular (3) - G3; e 28 leitos na clínica médica (12), cirúrgica (14) e cardiologia (02) - F2.

A população da pesquisa foi constituída dos pacientes internados nas unidades mencionadas, no período de agosto a setembro de 2015. A amostra foi definida em consonância aos seguintes critérios de elegibilidade: pacientes com mais de 18 anos, com orientação preservada no tempo e no espaço (este critério foi avaliado pela própria pesquisadora), ou presença de acompanhante/responsável aos menores de 18 anos e/ou àqueles com condições clínicas desfavoráveis à resposta e orientação no tempo e no espaço.

Os dados foram coletados por meio da aplicação de um instrumento denominado "Instrumento de Registro de Busca Ativa", que investiga a qualidade dos cuidados de enfermagem, elaborado e validado em outro hospital do sul do Brasil(13). O uso do instrumento se deu em consonância aos objetivos da pesquisa, ou seja, pela extração das variáveis que contemplam os indicadores relacionados à identificação do paciente (no leito e por pulseira) e à prevenção de quedas por meio da presença de grades no leito de pacientes, segundo risco.

Para avaliar o risco de quedas do leito utilizou-se a Morse Fall Scale, na sua versão traduzida e validada para o português ${ }^{(10)}$, constituída dos seguintes critérios de avaliação: histórico de quedas, diagnóstico secundário, auxílio na deambulação, terapia endovenosa/dispositivo endovenoso salinizado ou heparinizado, marcha e estado mental.

Cada um dos critérios anteriormente citados ao ser avaliado recebeu uma pontuação que varia de zero a 30 pontos, totalizando um escore de risco final a partir da soma de todas as pontuações dos respectivos critérios, a saber: risco baixo, de $0-24$; risco médio, de $25-44$ e risco alto, $\geq 45^{(10)}$. $\mathrm{Na}$ mensuração do indicador, foram considerados os pacientes classificados como médio e alto risco para verificar a presença de grades no leito, conforme o referencial metodológico escolhido estabelece ${ }^{(13)}$.

Realizou-se o teste piloto no mês de agosto, com seis pacientes selecionados aleatoriamente, para verificar possíveis problemas no uso do instrumento, o que não foi observado. Após isso, procedeu-se a observação dos pacientes, bem como o preenchimento dos itens do instrumento, durante 15 dias aleatórios nos meses de agosto e setembro de 2015, indo além do que orienta o referencial adotado, o qual estabelece a observação de 14 dias determinados casualmente ${ }^{(13)}$. A coleta foi integralmente procedida por uma única pesquisadora a fim de atenuar os vieses comuns aos estudos observacionais.

A análise foi realizada por meio do cálculo dos próprios indicadores mensurados, de acordo com as fórmulas recomendadas pelo instrumento utilizado(13). Posteriormente, a fim de classificar a qualidade ilustrada por cada indicador, os dados foram analisados de acordo com o Índice de Positividade (IP), que se refere ao percentual de respostas positivas, classificadas em: Assistência desejável $(100 \%$ de positividade); Assistência adequada (90 a 99\% de positividade); Assistência Segura (80 a $89 \%$ de 
positividade); Assistência limítrofe (71 a 79\% de positividade); e Assistência sofrível (Inferior a 70\% de positividade) $)^{(14)}$.

Ressalta-se que todas as etapas analíticas, bem como toda a análise estatística descritiva, foram procedidas após sumarização das informações em um banco de dados eletrônico do software Microsoft Office Excel 2010.

O projeto de pesquisa que fomentou o estudo foi submetido e aprovado pelo Comitê de Ética em Pesquisa da Universidade Estadual do Oeste do Paraná, com parecer sob protocolo 180.282/2015.

\section{RESULTADOS}

Ao todo, foram realizadas 1068 observações, o que correspondeu a 74,68\% da população internada no período, ou seja, os que atendiam aos critérios de elegibilidade. As demais não atendiam aos critérios ou recusaram-se. Destarte, a Tabela 1 sumariza os resultados obtidos à análise dos indicadores de interesse.

O Quadro 1 ilustra os resultados em relação à classificação da qualidade da assistência de enfermagem mediante os indicadores avaliados, por sua positividade.

Tabela 1 - Avaliação da conformidade dos indicadores relacionados à identificação do paciente e presença de grades no leito na prevenção de quedas. Cascavel, PR, Brasil, 2015

\begin{tabular}{lcccc} 
Indicador & \multicolumn{2}{c}{ Conforme } & \multicolumn{2}{c}{ Não Conforme } \\
\cline { 2 - 5 } & $\mathbf{n}$ & $\mathbf{\%}$ & $\mathbf{n}$ & $\mathbf{\%}$ \\
\hline Identificação do Leito do Paciente & 0 & 0 & 1068 & 100 \\
\hline Pacientes com Pulseira de Identificação & 818 & 76,6 & 250 & 23,4 \\
\hline Presença de Grades no Leito* & 782 & 95,48 & 37 & 4,52
\end{tabular}

*Conformidade calculada para pacientes com risco para quedas médio e alto, segundo avaliação pela Escala de Morse.

Quadro 1 - Qualidade da assistência ilustrada pelos indicadores avaliados, segundo Índice de Positividade. Cascavel, PR, Brasil, 2015

\begin{tabular}{|l|c|c|}
\hline Indicador & Índice de positividade (\%) & Qualidade da Assistência \\
\hline Identificação do leito do paciente & 0 & Assistência sofrível \\
\hline Pacientes com pulseira de identificação & 76,6 & Assistência limítrofe \\
\hline Presença de grades no leito & 95,48 & Assistência adequada \\
\hline
\end{tabular}

\section{- DISCUSSÃO}

Na Tabela 1 pode-se observar a (in) conformidade de cada indicador avaliado. Chama a atenção o fato de que a identificação do leito do paciente não ocorreu em nenhum sítio de observação, o que sinaliza que isso não é uma prática adotada pela instituição em sua rotina, que parece ter aderido - com eficácia limitada - à identificação por meio da pulseira. Destarte, esse achado difere ao que foi encontrado no estudo de validação do instrumento utilizado, no qual o campo de investigação, situado também no estado do Paraná, adotava a identificação do leito como norma organizacional. Este fato pode ter direcionado o mesmo indicador a uma conformidade determinada em $95,8 \%{ }^{(13)}$.

O dado antes exposto tem relevância porque, à prática clínica, sabe-se que há condições inerentes ao paciente que podem desfavorecer o correto posicionamento e/ou adesão da pulseira de identificação, tais como edema de membros e/ou generalizado, sudorese excessiva, amputação de membros, nível de 
consciência rebaixado, excesso de dispositivos assistenciais, entre outros. Deste modo, a identificação do paciente por meio do leito poderia ser uma medida que eliminasse a ausência de identificação, quando na impossibilidade da mesma ser procedida por meio de pulseira, pensamento que coaduna aos ditames expressos por órgão que rege a enfermagem em prol da segurança do paciente no Brasil(15).

Em relação à identificação do paciente por meio da pulseira, nota-se que houve conformidade expressivamente maior, quando se comparando o mesmo procedimento feito pela do leito (Tabela 1). Apesar disso, a identificação do cliente por pulseira também não alcançou nível de qualidade da assistência considerado satisfatório (Quadro 1). Essa afirmação ganha ainda mais importância quando aliada a dados semelhantes encontrados em um estudo realizado em outro hospital público de ensino, situado em Ribeirão Preto (SP), no qual a qualidade da assistência na identificação do paciente por pulseira foi classificada da mesma forma, ou seja, limítrofe ${ }^{(16)}$.

Cabe lembrar que, em abrangência nacional, há recomendação que a identificação de todos os pacientes deve ser realizada em sua admissão (permanecendo durante toda a internação) através da pulseira; bem como o serviço deverá definir como identificar pacientes que não possam utilizar este dispositivo(17), o que, novamente, reforça a perspectiva essencialmente negativa à análise do indicador de identificação no leito, que poderia ser uma alternativa para os 250 casos não identificados com pulseira (Tabela 1) tampouco à cabeceira do leito hospitalar, portanto, possivelmente mais expostos aos erros e, consequentemente, eventos adversos.

No que tange ao uso da pulseira de identificação, a instituição deverá utilizar no mínimo dois identificadores, tais como: nome completo do paciente, nome completo da mãe (no caso de recémnascidos), data de nascimento, e/ou número de prontuário(17). Estes aspectos não foram avaliados porque ultrapassam o que se propôs nesta pesquisa, mas sem dúvida são fatores que merecem atenção pelas lideranças hospitalares e também futuras investigações.

Diante dos resultados obtidos e a literatura consultada, converge-se à reflexão de que a adesão aos protocolos e medidas de segurança do paciente, tais como a identificação do paciente por pulseira, não deve seguir a lógica não rara de consumo acrítico dos conhecimentos científicos, mas sim ser um aporte à cultura organizacional sistêmica, em prol da qualidade e da segurança, o que ainda é um desafio a ser enfrentado, em virtude de ainda existir persistência da cultura contrária à melhoria contínua no setor hospitalar ${ }^{(3,18)}$.

Corrobora ao explicitado anteriormente, a menção de que apenas o "mero" uso da pulseira pelo paciente (ou seja, uso acrítico) não garante a sua segurança, uma vez que os profissionais devem conferi-la antes da realização de qualquer procedimento, a fim de evitar os erros e culminar à finalidade da pulseira, qual seja: aporte à segurança do paciente ${ }^{(11)}$. Neste sentido, autores ${ }^{(7)}$ afirmaram recentemente que no cotidiano do trabalho a prática de verificação das pulseiras acaba negligenciada pelos profissionais, sobretudo dos pacientes com longa internação; com isso, esses recomendam que haja cooperação mútua entre profissionais, usuários e gestores de saúde, a fim de consolidar a identificação como alavanca à segurança do paciente e não aporte burocrático na organização ${ }^{(7)}$.

Antagonicamente aos dados apresentados e debatidos, percebe-se que o indicador avaliado no que concerne à presença de grades no leito para pacientes classificados como médio ou alto risco para quedas, segundo a Escala de Morse ${ }^{(10)}$, obteve conformidade satisfatória, o que, segundo o referencial adotado, ilustrou a qualidade da assistência neste aspecto como adequada (Quadro 1). Não menos importante, este dado é também ratificado por outra investigação realizada em hospital privado, que, ao uso do mesmo referencial metodológico, classificou a qualidade vislumbrada pelo indicador do mesmo modo $^{(19)}$.

Reconhece-se, no entanto, que apesar de este achado ser notoriamente louvável, esta é, possivelmente, uma perspectiva que se limita à estrutura da organização hospitalar investigada, visto que o que foi mensurado diz respeito apenas à presença/ausência de grades nas camas do hospital, conforme orienta o instrumento de coleta e parte da análise dos dados ${ }^{(13)}$.

Por este motivo, cumpre refletir que a presença da grade no leito por si só não basta à prevenção da queda, visto que o posicionamento desta à sua elevação deve ser um processo contínuo de militância na busca pela segurança do paciente, além de outras medidas igualmente importantes, como: orientação de familiares; cuidados ambientais (umidade do piso, posicionamento de mobiliário, pertences 
próximos ao paciente, entre outros); avaliação neurológica; manutenção de campainha próxima ao paciente, se na presença desta; entre outros ${ }^{(20)}$.

Os cuidados acima descritos são importantes e necessários porque a evidência do risco associado à assistência é mais acentuada no ambiente hospitalar ${ }^{(3,18)}$, fato que posiciona a prevenção da queda como um elemento de importância crucial à promoção da segurança do paciente, especialmente à vigília constante e preventiva da equipe de enfermagem, que, sabidamente, é a única categoria profissional a acompanhar o paciente hospitalizado nas 24 horas do dia.

Nesta direção, pesquisadores alvitram que, além de cuidados intrínsecos ao paciente, a prevenção da queda perpassa por questões inerentes ao próprio capital humano da enfermagem, ao exemplo do adequado dimensionamento de pessoal, que corresponde ao quali-quantitativo ajustado para suprir a demanda de cuidados de forma qualificada e segura ${ }^{(21)}$.

Reforça o pressuposto anterior um estudo recente realizado em um hospital de grande porte de Porto Alegre, o qual evidenciou que, ao ser exposta a elevada carga de trabalho - a qual pode ser associada ao déficit do quantitativo de pessoal -, a equipe de enfermagem apresentou indicadores de desempenho igualmente deficitários, entre eles, o aumento das taxas de queda do leito ${ }^{(22)}$.

Cabe aludir, ainda, que a prevenção da queda possivelmente se inicia pela detecção do risco à ocorrência deste evento indesejável e potencialmente danoso ${ }^{(9,10)}$. No entanto, relembra-se a necessidade de utilizar os instrumentos/ferramentas de organização do cuidado, tal como as escalas de risco para queda ${ }^{(10)}$, de forma racional e crítica. Por isso, alvitra-se que o papel do enfermeiro é salientado, já que durante sua formação é preparado para desenvolver metodologias que propiciem análise do processo assistencial objetivando o atendimento das expectativas e necessidades dos pacientes, e as atividades de controle de qualidade proporcionam a estes profissionais uma contínua avaliação da prática assistencial na busca de propiciar excelência no cuidado de enfermagem ${ }^{(23)}$.

No tocante ao explicitado, fica evidente que a participação da equipe de enfermagem na prevenção da queda no ambiente hospitalar é fundamental, e, de acordo com os resultados encontrados, ainda que seja apenas uma perspectiva pontual, há de se convir que a organização alcançou parâmetros satisfatórios de qualidade.

Todavia, reafirma-se a necessidade de investigar os aspectos que ultrapassam a estrutura dos leitos, possibilitando a análise do processo de cuidar propriamente dito, ou seja, as medidas preditivas à prevenção da queda viabilizadas pela enfermagem, sugestão que vale também para a verificação e controle de uso de pulseiras e outros possíveis dispositivos de identificação do paciente.

\section{CONCLUSÃO}

Através deste estudo, foi possível avaliar indicadores de interesse à segurança do paciente, e, consequentemente, fatores que influenciam a qualidade do cuidado. Neste sentido, os resultados dos indicadores ilustraram qualidade multifacetada, em virtude de que houve sua classificação tanto em aspecto satisfatório (grades no leito), quanto insatisfatórios, a respeito da identificação do paciente por pulseira e, principalmente, através do leito.

Com base nos resultados, conclui-se que a organização investigada carece de investimentos à adesão eficaz da identificação do paciente. Uma sugestão para melhoria dessa problemática é o empoderamento do enfermeiro em relação à tomada de decisão para identificação do paciente conforme sua necessidade, ou seja, escolha racional.

Apesar da perspectiva favorável à prevenção da queda identificada, reafirma-se a necessidade de se investigar o processo de cuidado no bojo deste bem, haja vista a importância que o mesmo carrega à promoção da segurança. Ademais, em virtude das limitações expressas à pesquisa, como seu recorte transversal e a ausência de análise estatística inferencial, espera-se que novas pesquisas sejam fomentadas, especialmente no que diz respeito ao uso racional e crítico da identificação do paciente, e também dos cuidados relacionados à prevenção das quedas. 
1. Pena MM, da Silva EMS, Tronchin DMR, Melleiro MM. The use of the quality model of Parasuraman, Zeithaml and Berry in health services. Rev. esc. Enferm. USP. [Internet] 2013; 47(5) [acesso em 03 ago 2015]. Disponível: http://dx.doi.org/10.1590/S0080-623420130000500030.

2. World Health Organization (WHO) [Internet]. Quality of care: a process for making strategic choices in health systems. Geneva; 2006 [acesso em 03 ago 2015]. Disponível:

http://www.who.int/management/quality/assurance/QualityCare_B.Def.pdf

3. Silva BEMS, Melleiro MM. Patient safety culture in a teaching hospital: differences in perception existing in the different scenarios of this institution. Texto contexto Enferm. [Internet] 2015; 24(2) [acesso em 16 out 2015]. Disponível: http://dx.doi.org/10.1590/0104-07072015000192014.

4. Ministério da Saúde (BR). Portaria n. 529, de 1 o de abril de 2013. Institui o Programa Nacional de Segurança do Paciente. Brasília; 2013. [acesso em 08 ago 2015]. Disponível:

http://bvsms.saude.gov.br/bvs/saudelegis/gm/2013/prt0529_01_04_2013.html

5. Abreu C, Mendes A, Monteiro J, Santos FR. Falls in hospital settings: a longitudinal study. Rev. Latino-Am. Enfermagem. [Internet] 2012; 20(3) [acesso em 01 de nov 2015]. Disponível:

http://dx.doi.org/10.1590/S0104-11692012000300023

6. Severo IM, Almeida MA, Kuchenbecker R, Vieira DFVB, Weschenfelder ME, Pinto L RC, et al. Risk factors for falls in hospitalized adult patients: an integrative review. Rev Esc enferm USP. [Internet] 2014; 48(3) [acesso em 01 nov 2015]. Disponível: http://dx.doi.org/10.1590/S0080-623420140000300021

7. Tase TH, Lourenção DCA, Bianchini SM, Tronchin DMR. Patient identification in healthcare organizations: an emerging debate. Rev Gaúcha Enferm. [Internet] 2013; 34(2). [acesso em 01 nov 2015]. Disponível:

http://dx.doi.org/10.1590/S1983-14472013000300025.

8. World Health Organization (WHO), Joint Comission Resources, Joint Comission International. Patient safety Solutions. [Internet] 2007; 1(8) [acesso em 16 out 2015]. Disponível:

http://www.who.int/patientsafety/solutions/patientsafety/PS-Solution2.pdf

9. da Costa-Dias MJM, Ferreira, PL. Escalas de avaliação de risco de quedas. Revista de Enfermagem Referência. [Internet] 2014; 4(2) [acesso em 01 nov 2015]. Disponível: http://ui.esenfc.pt/rr/index.php?module=rr\&target=pub licationDetails\&pesquisa=\&id_artigo=2456\&id_revista=24\&id_edicao=66

10. Urbanetto JS, Creutzberg M, Franz F, Ojeda BS, Gustavo AS, Bittencourt HR, et al. Morse fall scale: translation and transcultural adaptation for the portuguese language. Rev. esc. enferm. USP. [Internet] 2013; 47(3) [acesso em 01 nov 2015]. Disponível: http://dx.doi.org/10.1590/S0080-623420130000300007

11. Hoffmeister LV, de Moura GMSS. Use of identification wristbands among patients receiving inpatient treatment in a teaching hospital. Rev. Latino-Am. Enfermagem. [Internet] 2015; 23(1) [acesso em 01 nov 2015]. Disponível: http://dx.doi.org/10.1590/0104-1169.0144.2522

12. Vituri DW, Évora YDM. Reliability of indicators of nursing care quality: testing interexaminer agreement and reliability. Rev. Latino-Am. Enfermagem. [Internet] 2014; 22(2) [acesso em 01 nov 2015]. Disponível:

http://dx.doi.org/10.1590/0104-1169.3262.2407

13. Vituri DW. Desenvolvimento e validação de um instrumento para avaliação da qualidade do cuidado de enfermagem [dissertação]. Maringá (PR): Universidade Estadual de Maringá; 2007.

14. Haddad MCLF. Qualidade da assistência de enfermagem: processo de avaliação em hospital universitário público [tese]. Ribeirão Preto (SP): Universidade de São Paulo; 2004.

15. Rede Brasileira de Enfermagem e Segurança do Paciente (REBRAENSP). Estratégias para a segurança do paciente: manual para profissionais da saúde. Porto Alegre: EDIPUCRS; 2013.

16. Caldana G, Gabriel CS, Bernardes A, de Pádua RX, Vituri DW, Rossaneis MA. Avaliação da qualidade de cuidados de enfermagem em hospital público. Semina: Ciências Biológicas e da Saúde. [Internet] 2013; 34(2) 
[acesso em 10 out 2015]. Disponível: http://dx.doi.org/10.5433/1679-0367.2013v34n2p187

17. Ministério da Saúde (BR). Protocolo de identificação do paciente. Brasília, 2013 [acesso em 01 ago 2015]. Disponível: http://proqualis.net/sites/proqualis.net/files/Protocolo\%20de\%20Identifica $\%$ C3\%A7\%C3\%A3o\%20 do\%20Paciente.pdf

18. de Souza VS, Kawamoto AM, Oliveira JLC, Tonini NS, Fernandes LM, Nicola AL. Errors and adverse events: the interface with health professionals' safety culture. Cogitare enferm. [Internet] 2015; 20(3) [acesso em 01 nov 2015]. Disponível: http://dx.doi.org/10.5380/ce.v20i3.40687

19. Caldana G, Gabriel CS, Ocha FLR, Bernardes A, Françolin L, da Costa DB. Avaliação da qualidade de cuidados de enfermagem em hospital privado. Rev. Eletr. Enf [Internet]. 2013; 15(4) [acesso em 18 out 2015]. Disponível: http://dx.doi.org/10.5216/ree.v15i4.19655

20. Luzia MF, Almeida MA, Lucena AF. Nursing care mapping for patients at risk of falls in the nursing interventions classification. Rev. esc. enferm. USP. [Internet] 2014; 48(4) [acesso em 01 nov 2015]. Disponível: http://dx.doi.org/10.1590/S0080-623420140000400009

21. Inoue KC, Matsuda LM, de Melo WA, Murassaki ACY, Hayakawa LY. Risco de queda da cama. O desafio da enfermagem para a segurança do paciente. Invest Educ Enferm. [Internet] 2011; 29(3) [acesso em 01 nov 2015]. Disponível: https://aprendeenlinea.udea.edu.co/revistas/index.php/iee/article/view/8679/9820

22. Magalhães AMM, Dall'Agnol CM, Marck PB. Nursing workload and patient safety - a mixed method study with an ecological restorative approach. Rev. Latino-Am. Enfermagem. [Internet] 2013; 21(spe) [acesso em 01 nov 2015]. Disponível: http://dx.doi.org/10.1590/S0104-11692013000700019

23. Franco JN, Barros BPA, Vaidotas M, D'Innocenzo M. Percepção dos enfermeiros sobre os resultados dos indicadores de qualidade na melhoria da prática assistencial. Rev. bras. enferm. [Internet] 2010; 63(5) [acesso em 30 dez 2015]. Disponível: http://dx.doi.org/10.1590/S0034-71672010000500018 